\title{
MODEL PREDICTIVE CONTROL FOR A CLASS OF HYBRID SYSTEMS
}

\author{
Olav Slupphaug and Bjarne A. Foss \\ Norwegian University of Science and Technology \\ Department of Engineering Cybernetics \\ Fax : +4773594399 and e-mail: baf@itk.ntnu.no
}

Keywords : Model predictive control, hybrid systems, sampled-data control, stability.

\begin{abstract}
In this paper we investigate the use of model predictive control (MPC) as a means for controlling a class of sampled-data hybrid systems. A weak form of uniform asymptotical stability is established for the closed-loop. This is done by first establishing stability results for an associated discrete-time system. Conditions for existence of a solution to the underlying optimization problem are also provided.
\end{abstract}

\section{Introduction}

Industrial control systems are generally comprised of logic and continuous controllers. The logic performs functions like starting process equipment, controlling low-level (or regulatory) controllers, and controlling discrete control inputs (on/off valves, pumps etc.). The logic may for instance switch between different continuous controllers depending on the operating conditions of a process. Continuous controllers are used for regulatory control and for supervisory control. On the regulatory level PID-controllers constitute the typical continuous controllers. On the supervisory level continuous controllers compute the setpoints for the regulatory controllers using for instance an optimization algorithm. In our context it is important to note that the logic and continuous controllers in many cases are closely coupled, hence they form a hybrid dynamic system (HS). Typically, industrial design practice is based on a separate construction of the continuous and logic part of a control system. By this, the effect of the interaction between the continuous and logic part is difficult to foresee during the design-phase.

The hybrid nature of a system may also originate from the controlled system itself. In chemical process control a process may change its characteristics abruptly. One example of this is the change between laminar and turbulent flow conditions.

The above observation forms the motivation for this study in which we investigate the design of hybrid control systems. We utilize optimization-based control, or more specifically, MPC for this purpose. This is done by including both continuous- and discrete control inputs as decision variables in the optimization. Further, the switching instants of the control inputs are also decision variables.

There are extensive research efforts in HS today. Research is conducted in both mathematical-, control-, and computer science communities, see eg. [1] and references therein. Some of this research is directed towards process control as reported in [2].

MPC has been an active research area for close to two decades. The research has been driven by numerous successful applications of the technology, and during the last years a sound theoretical foundation has been established [3].

This paper consists of two parts. First, the proposed control strategy is presented and certain aspects of it are discussed. Second, we present stability results for our proposed control strategy.

In what follows $:=(=:)$ denotes assignment, a function $f: \mathbb{X} \times \ldots \times \mathbb{Y} \rightarrow \mathbb{W}$ is denoted $f(\cdot, \ldots, \cdot), \mathbb{N}:=\{0,1, \ldots\}$, $\mathbb{N}^{+}:=\{1, \ldots\}, I_{N}:=\{0, \ldots, N\}$, and $I_{N}^{+}:=\{1, \ldots, N\}$.

\section{Problem formulation}

We have tried to keep the notational complexity as low as possible. Further the symbolism is, as far as possible, made consistent with mainstream work in the MPC area.

\subsection{The Plant}

The plant to be controlled is assumed to be known and described by the following continuous-time, time-invariant constrained linear system:

$$
\begin{aligned}
\Sigma: \quad \frac{\mathrm{d}}{\mathrm{dt}} x(t) & =: \dot{x}(t)=A x(t)+B u(t), t \geq 0, \\
x(0) & - \text { given, } \\
n & :=\operatorname{dim}(x(t)) ; m:=\operatorname{dim}(u(t)), \\
x(t) & \in X \subseteq R^{n} ; u(t) \in U \subseteq R^{m}, \\
A & \in R^{n \times n} ; B \in R^{n \times m},
\end{aligned}
$$

where $x(t)$ is the state of $\Sigma$ at time $t$, and $u(t)$ is its control input at time $t$. We have available a sampled state measurement. We assume that the state and control input of $\Sigma$ are chosen so that the control objective is to bring the state to the origin. Thus, naturally we let $0 \in X \times U$. Also, $X$ and $U$ are assumed to be closed.

\subsection{The Controller}

As mentioned in the introduction, an MPC strategy is used to design the controller. Thus, as a part of the controller, a predictor is needed.

\subsubsection{Predictor}

We are considering the nominal case, and use the sampled solution of $\Sigma$ as the predictor:

$$
\begin{aligned}
z(\tau ; \pi) & :=e^{A \tau} z_{0}+\int_{0}^{\tau} e^{A(\tau-\sigma)} B v(\sigma ; \pi) \mathrm{d} \sigma \\
z_{j} & :=z(j \Delta ; \pi)
\end{aligned}
$$

where 
$\Delta$ the sample period $(\Delta>0)$.

Let $k \geq 0$ be the current discrete-time index, then

$t_{k}:=k \Delta$ the current time;

$j$ a generic future (relative to $k$ ) discrete-time index;

$z_{0}$ in general some estimate of $x\left(t_{k}\right)$. In this study $z_{0}=x\left(t_{k}\right)$;

$z(\tau ; \pi)$ the prediction of the state of $\Sigma$ at time $t_{k}+\tau$ using $\pi$ (see below);

$z_{j}$ the prediction of the sampled state at time $t_{k}+j \Delta$ using $\pi$

$v(\sigma ; \pi)$ the open-loop control input at time $t_{k}+\sigma$, induced by $\pi$. Cf. Eq. (2).

Further, we define the following symbols:

$T_{M}$ denotes both the finite move horizon and the endpoint of the move horizon (the open-loop control input is identically zero beyond this horizon);

$M$ the (maximum) allowable number of control input switches on the move-horizon;

$\pi_{\tau}:=\left(\tau_{i}\right)_{i \in I_{M}^{+}}$where $\tau_{i} \in\left[0, T_{M}\right] \wedge \tau_{i} \leq \tau_{i+1}$, the open-loop timing sequence;

$\tau_{0}:=0$

$\tau_{M+1}:=T_{M}$

$\pi_{v}:=\left(v_{i}\right)_{i \in I_{M-1}}$ where $v_{i} \in U$, the open-loop control input sequence;

$v_{i}, i \in I_{M-1}$ the open-loop control input at the timeinterval $\left[t_{k}+\tau_{i}, t_{k}+\tau_{i+1}\right)$;

$\tau_{i}, i \in I_{M}^{+}$instants at which the open-loop control input is switched.

At last,

$\pi:=\left(\pi_{\tau}, \pi_{v}\right)$ the timed open-loop control input sequence.

The open-loop control input, $v(\cdot ; \cdot)$, used in the predictor, Eqs. (1), is defined as:

$$
v(\sigma ; \pi):= \begin{cases}v_{i}, & \sigma \in\left[\tau_{i}, \tau_{i+1}\right), i \in I_{M-1}, \\ 0, & \sigma \geq \tau_{M} .\end{cases}
$$

2.2.2 Performance index

The performance index is defined as:

$$
\phi\left(\pi ; x_{k}, k\right):=\sum_{j \in I_{N-1}} L_{z, k+j}\left(z_{j}\right)
$$

$$
+\sum_{i \in I_{M-1}} L_{v, i}^{k}\left(\pi_{\tau}, v_{i}\right)
$$

where

$L_{z, j}(\cdot), j \in \mathbb{N}$ nonnegative real functions,

$L_{v, i}^{k}(\cdot, \cdot),(k, i) \in \mathbb{N} \times I_{M-1}$ nonnegative real functions,

$$
x_{k}:=x\left(t_{k}\right),
$$

$N \Delta$ the prediction horizon.

The notation $L_{v, i}^{k}(\cdot, \cdot)$ is necessary since $L_{v, i+k}(\cdot, \cdot)$ do not, in the present approach, provide the desired flexibility. Note that, of course, $L_{v, k+(i+1)}(\cdot, \cdot)=L_{v,(k+1)+i}(\cdot, \cdot)$. We, however, would like to avoid this forced equality, and, indeed, $L_{v, i+1}^{k}(\cdot, \cdot)$ and $L_{v, i}^{k+1}(\cdot, \cdot)$ may be chosen independent of each other. A typical choice for $L_{v, i}^{k}(\cdot, \cdot)$ would be $L_{v, i}^{k}\left(\pi_{\tau}, v_{i}\right)=\left(\tau_{i+1}-\tau_{i}\right) / T_{M} \tilde{L}_{k+i}\left(v_{i}\right)$, where $\tilde{L}_{j}(\cdot), \forall j \in \mathbb{N}$, are nonnegative real functions.

\subsubsection{Optimization}

Let

$$
\begin{aligned}
& \Pi_{\tau}:=\left\{\pi_{\tau}: \pi_{\tau} \text { satisfies its definition }\right\} \\
& \Pi_{v}:=\left\{\pi_{v}: \pi_{v} \text { satisfies its definition }\right\}
\end{aligned}
$$

We define the set of all possible timed open-loop control input sequences by

$$
\Pi:=\Pi_{\tau} \times \Pi_{v} .
$$

The optimal timed open-loop control input sequence at time $t_{k}$ is given by the solution to the minimization problem, $\mathcal{P}\left(x_{k}, k\right)$,

$$
\begin{aligned}
\mathcal{P}\left(x_{k}, k\right): & \min _{\pi \in \Pi} \phi\left(\pi ; x_{k}, k\right) \\
\text { s.t. } & \text { Eqs. }(1), \\
& z_{i} \in X, \forall i \in I_{N-1}^{+}, \\
& z_{N}=0 .
\end{aligned}
$$

The final state equality constraint, $z_{N}=0$, is present for closed-loop stability reasons.

We denote the minimizer $\pi_{k}^{*}\left(x_{k}\right)$, i.e.

$$
\pi_{k}^{*}\left(x_{k}\right):=\arg \mathcal{P}\left(x_{k}, k\right) .
$$

Let

$$
\phi^{*}\left(x_{k}, k\right):=\phi\left(\pi_{k}^{*}\left(x_{k}\right) ; x_{k}, k\right) .
$$

We recursively define

$$
\begin{aligned}
\pi_{k}^{*}\left(x_{k}\right) & =:\left(\pi_{\tau, k}^{*}\left(x_{k}\right), \pi_{v, k}^{*}\left(x_{k}\right)\right) \\
& =:\left(\left(\tau_{k, i}^{*}\left(x_{k}\right)\right)_{i \in I_{M}^{+}},\left(v_{k, i}^{*}\left(x_{k}\right)\right)_{i \in I_{M-1}}\right) .
\end{aligned}
$$


With the solution of $\mathcal{P}\left(x_{k}, k\right)$ we also associate $v_{k}^{*}(\cdot ; \cdot)$, $z_{k}^{*}(\cdot ; \cdot)$, and $z_{k, j}^{*}(\cdot)$, where

$$
\begin{aligned}
v_{k}^{*}\left(\tau ; x_{k}\right) & :=v\left(\tau ; \pi_{k}^{*}\left(x_{k}\right)\right), \\
z_{k}^{*}\left(\tau ; x_{k}\right) & :=z\left(\tau ; \pi_{k}^{*}\left(x_{k}\right)\right), \\
z_{k, j}^{*}\left(x_{k}\right) & :=z_{k}^{*}\left(j \Delta ; x_{k}\right) .
\end{aligned}
$$

The control input at the time-span $\left[t_{k}, t_{k+1}\right)$ is by definition of MPC

$$
u(t):=v_{k}^{*}\left(t-t_{k} ; x_{k}\right), \quad t \in\left[t_{k}, t_{k+1}\right) .
$$

Observe that sampled state feedback is present. Signals and symbols are depicted in Fig. 1.

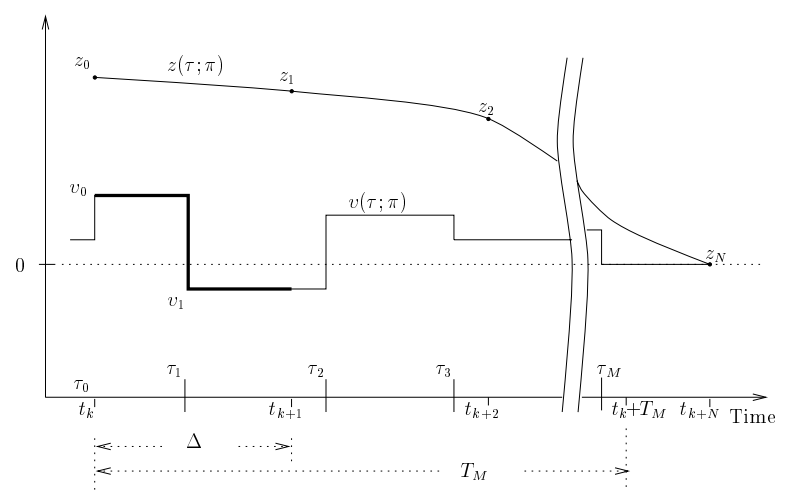

Figure 1: The control input $v(\cdot ; \cdot)$ switches on the continuum $\left[t_{k}, t_{k}+T_{M}\right]$. The switching times are denoted $\tau_{i}$. The continuous-time system is sampled at equidistant time-instants with sample period $\Delta$, as indicated by $\left(t_{k+j}, z_{j}\right)$. The control input returns to zero at $\tau_{M}$. If the depicted $v(\cdot ; \cdot)$ is the optimizing $v_{k}^{*}(\cdot ; \cdot)$, then the "fat" part of $v(\cdot ; \cdot)$ would represent $u(t), t \in\left[t_{k}, t_{k+1}\right)$.

\subsection{Discussion}

We allow $U$ to be formed by a Cartesian product of unions of closed connected sets (associated with the continuous control inputs) and finite sets (associated with the discrete control inputs). Note that such sets will always be disconnected (and nonconvex), as opposed to most work on model predictive control where it is assumed that $U$ is at least connected, in fact $U$ is almost always assumed to be convex. Convex $X$ and $U$ combined with a predictor/performance index pair giving a convex optimization problem lead to readily computable optimal solutions. In our case, however, $\mathcal{P}\left(x_{k}, k\right)$ becomes a nonlinear mixeddiscrete programming problem when there are discrete valued elements in $u(t)$. Even if the performance index is a quadratic form, the system is linear, and $u(\cdot)$ takes on values in some convex set, $\mathcal{P}\left(x_{k}, k\right)$ is in general a nonconvex programming problem due to the predictor's nonlinear dependence on the $\tau_{i}$ 's.

The controller is assumed to receive the current state of the plant at known time-instants, $t_{k}$, further, we assume it can change the control input at any time-instant, limited, however, to a finite number of times, $M$, on a finite time interval, $T_{M}$. This assumption makes our approach different from both pure discrete-time and continuous-time MPC approaches. We let the time-instants at which we obtain information be equidistant, and the time-span between each instant equals the sample period, $\Delta$. It is also assumed that the quantization of $x(t)$ and $u(t)$ is infinitely fine.

Our choice of parameterization of the open-loop control input, cf. Eq. (2), induce a piecewise constant closed-loop control input. There are, of course, also other sensible parameterizations. However, discrete control inputs are easily included in our choice, further it is the simplest parameterization possible, hence it does not complicate the analysis more than necessary.

In our case we may have an infinite or finite prediction horizon combined with a move-horizon restricted to be finite with a finite number of moves on it. In addition the number of moves, $M$, and the number of stages, $N$, on the prediction horizon is not necessarily equal in the finite case (certainly not in the infinite case), and either one can be the larger. However, the move horizon cannot be longer than the prediction horizon $\left(T_{M} \leq N \Delta\right)$. The control input is not restricted to switch only at the sample instants, $t_{k}$ - the switching times, $\tau_{i}$, are decision variables. In particular this latter point does not allow an application of the previously reported results known to the authors. Neither the predictor nor the performance index may be cast into the general discrete-time framework of Keerthi and Gilbert [4].

As is seen from the above discussion, there are several differences between our approach and others. However, we note that if we drop the switching times as decision variables and instead fix $\pi_{\tau}=\pi_{\tau}^{\Delta}:=(\Delta, \ldots, M \Delta)$, then convex $X$ and $U$, and quadratic forms as stage-costs give a "standard" linear MPC formulation, apart from the sampled-data formulation.

\section{Stability analysis of the pro- posed MPC approach}

Several MPC approaches which ensure nominal closedloop stability have been proposed. Cf. [5] for a review. In our work we will use the line of reasoning used in [4] as a basis, and also we will use ideas from [6].

Let $R_{+}$denote the set of nonnegative reals. $\|\cdot\|$ denotes the Eucledian norm. For $\varepsilon \in R_{+}, N(\varepsilon):=\{x:\|x\| \leq \varepsilon\}$. A function $W: R_{+} \rightarrow R_{+}$is said to belong to class $\mathcal{K}_{0}$ if: (a) it is continuous; (b) $W(s)=0 \Leftrightarrow s=0$. W(.) is in class $\mathcal{K}_{+}$if $W(\cdot) \in \mathcal{K}_{0}$ and is strictly increasing. $W(\cdot)$ is in class $\mathcal{K}_{\infty}$ if $W(\cdot) \in \mathcal{K}_{+}$and $W(s) \rightarrow \infty$ when $s \rightarrow \infty$. $\lfloor t\rfloor$ denotes the largest integer smaller than or equal to $t$, while $\lceil t\rceil$ denotes the smallest integer greater than or equal to $t$.

\subsection{Stability preliminaries}

The stability properties of the closed-loop will be established by using the following theorem with $\phi^{*}(\cdot, \cdot)$ as the 
Liapunov function $V(\cdot, \cdot)$.

Consider the constrained time-varying system

$$
x_{k+1}=f_{k}\left(x_{k}\right), \quad x_{k} \in \tilde{X} \subseteq R^{n}, \quad k \geq 0,
$$

where $f_{k}: \tilde{X} \rightarrow \tilde{X}$ for all $k \geq 0$. For $(l, a) \in \mathbb{N} \times \tilde{X}$, let $x_{k}(l, a), k \geq l$, denote the solution of (4), given $x_{l}=a$. A state $x_{e} \in R^{n}$ is said to be an equilibrium state for (4) if $x_{e} \in \tilde{X}$ and $f_{k}\left(x_{e}\right)=x_{e}$ for all $k \geq 0$. Assume that $x=0$ is an equilibrium state for (4).

\section{Theorem 1}

Suppose $\exists \lambda>0, V: \mathbb{N} \times N(\lambda) \rightarrow R, \alpha(\cdot) \in \mathcal{K}_{+}$, $\beta(\cdot) \in \mathcal{K}_{+}, \gamma(\cdot) \in \mathcal{K}_{+}$, which satisfy the following conditions: (a) $N(\lambda) \subset \tilde{X}$; (b) $\alpha(\|a\|) \leq V(l, a) \leq$ $\beta(\|a\|), \quad \forall(l, a) \in \mathbb{N} \times N(\lambda) ;$ and (c) $V(l, a)-V(l+$ $\left.1, x_{l+1}(l, a)\right) \geq \gamma(\|a\|), \forall(l, a) \in \mathbb{N} \times N(\lambda)$. Then for (4), the following result holds: $x=0$ is uniformly asymptotically stable (UAS).

The proof is omitted due to page limitation. It can be found in [7].

\subsection{Existence of a solution to $\mathcal{P}\left(x_{k}, k\right)$}

The stability results depend on solving $\mathcal{P}\left(x_{k}, k\right)$, hence at least sufficient conditions for this to be possible should be found.

Sufficient conditions for existence of solutions to discrete optimal control problems are also reported elsewhere, including [6], [8], and [9].

By a feasible timed open-loop control input sequence, it is meant a $\pi$ that satisfies the constraints imposed on $\mathcal{P}\left(x_{k}, k\right)$.

\section{Proposition 1}

If $L_{z, j}: R^{n} \rightarrow R, j \in \mathbb{N}$, and $L_{v, i}^{k}: \Pi_{\tau} \times R^{m} \rightarrow R,(k, i) \in$ $\mathbb{N} \times I_{M-1}$, is nonnegative lower semicontinuous; $\forall(k, i) \in$ $\mathbb{N} \times I_{M-1}, \pi_{\tau} \in \Pi_{\tau} L_{v, i}^{k}\left(\pi_{\tau}, v\right) \rightarrow \infty$ as $\|v\| \rightarrow \infty$; and $X$ and $U$ are closed, then the existence of a feasible timed open-loop control input sequence $\pi$ that yields a bounded performance index $\phi(\pi ; x, k)$ for initial condition $x$ implies the existence of an optimal timed open-loop control input sequence, $\pi_{k}^{*}(x)$, for this initial condition.

The proof is omitted due to page limitation. It can be found in [7].

\subsection{Stability results}

In what follows we assume the prediction horizon, $N$, is finite, contrary to the existence proposition where there was no such restriction.

The (continuous-time) closed-loop system is:

$$
\begin{aligned}
\Sigma_{C L}: \quad \dot{x}(t)=A x(t)+B v_{k}^{*}\left(t-t_{k} ; x\left(t_{k}\right)\right), & \\
x\left(t_{k}\right) & \in \tilde{X}_{F}, t \in\left[t_{k}, t_{k+1}\right), k \geq 0 .
\end{aligned}
$$

We also have the following associated discrete-time closedloop system $-\Sigma_{C L}$ as observed at the sampling instants:

$$
\begin{gathered}
\Sigma_{A C L}: \quad x_{k+1}=x\left(t_{k+1}\right)=z_{k}^{*}\left(\Delta ; \pi_{k}^{*}\left(x_{k}\right)\right)=z_{k, 1}^{*}\left(x_{k}\right), \\
x_{k} \in \tilde{X}_{F}, k \geq 0 .
\end{gathered}
$$

Below, $\tilde{X}_{F}$ is defined and shown to be nonempty.

We proceed by considering $\Sigma_{A C L}$. Let the solution of $\Sigma_{A C L}$ be denoted $x_{k}(l, a)$ for $k \geq l$ given $x_{l}=a$.

Similar to [4] we define a predictor property $\mathcal{C}_{F}$, which is implied by controllability (for interpretation, see immediately below) of the predictor, see [4]. Let $\pi_{\tau}=\pi_{\tau}^{\Delta}$, and delete the columns of the control distribution matrix corresponding to the discrete control inputs before performing the usual algebraic test for controllability on the resulting associated linear discrete-time system. In the nominal case considered here, controllability of the predictor follows from controllability of $\Sigma$ (in the sense described above) as long as we have a non-pathological sampling period with respect to $A[10]$.

\section{Definition 1 (Property $\mathcal{C}_{F}$ )}

The predictor (1) has property $\mathcal{C}_{F}$ if $\exists W(\cdot) \quad \in$ $\mathcal{K}_{\infty}, M^{\prime}, T_{M}^{\prime}, N^{\prime}$ such that for every $a \in R^{n}, \exists \pi \in$ $\Pi_{\tau}^{\prime} \times\left(R^{c} \times D_{1} \times \ldots \times D_{m-c}\right)^{M^{\prime}}$ such that for $z_{0}=a$ :

$$
z_{N^{\prime}}=0 \text { and } \sum_{i=0}^{M^{\prime}-1}\left\|v_{i}\right\|+\sum_{j=0}^{N^{\prime}-1}\left\|z_{j}\right\| \leq W\left(\left\|z_{0}\right\|\right),
$$

where $c$ is the number of continuous control inputs, $D_{i}$, $i \in I_{m-c}^{+}$, are the control input sets associated with the discrete control inputs, and $\Pi_{\tau}^{\prime}$ is a redefinition of $\Pi_{\tau}$ using $M^{\prime}$ and $T_{M}^{\prime}$ instead of, respectively, $M$ and $T_{M}$.

In addition to the predictor property and the assumptions made in the existence proposition, A1-A4 (see below), we need some additional assumptions on the functions comprising the performance index, and the constraints. (Note that A4 is a stronger version of the growth condition in the existence proposition.) For completeness we restate the previous assumptions together with the ones which are purely related to the stability question, A5-A10.

A1 $X$ and $U$ are closed.

A2 $\forall j \in \mathbb{N} L_{z, j}: R^{n} \rightarrow R$ nonnegative lower semicontinuous real functions.

A3 $\forall(k, i) \in \mathbb{N} \times I_{M-1} L_{v, i}^{k}: \Pi_{\tau} \times R^{m} \rightarrow R$ nonnegative lower semicontinuous real functions.

$\mathrm{A} 4 \forall(k, i) \in \mathbb{N} \times I_{M-1}, \pi_{\tau} \in \Pi_{\tau}, \exists H_{1}(\cdot) \in$ $\mathcal{K}_{\infty} L_{v, i}^{k}\left(\pi_{\tau}, v\right) \geq H_{1}(\|v\|)$.

A5 $\forall(k, i) \in \mathbb{N} \times I_{M-1}, \pi_{\tau} \in \Pi_{\tau}, \exists H_{2}(\cdot) \in$ $\mathcal{K}_{\infty} L_{v, i}^{k}\left(\pi_{\tau}, v\right) \leq H_{2}(\|v\|)$.

A6 $\forall j \in \mathbb{N}, x \in R^{n}, \exists H_{3}(\cdot) \in \mathcal{K}_{\infty} L_{z, j}(x) \leq H_{3}(\|x\|)$.

A7 $\forall j \in \mathbb{N}, x \in R^{n}, \exists H_{4}(\cdot) \in \mathcal{K}_{+} L_{z, j}(x) \geq H_{4}(\|x\|)$.

A8 $0 \in$ Interior $X \times U_{c}$, where $U_{c}$ denotes the Cartesian product of the $c$ control sets associated with the continuous control inputs.

A9 $M \geq M^{\prime}, T_{M} \geq T_{M}^{\prime}$, and $N \geq N^{\prime}$. 
A10 $\forall(k, i) \in \mathbb{N} \times I_{M-1},\left(\pi_{\tau}, \tilde{\pi}_{\tau}\right) \in \Pi_{\tau}^{2},\left(v_{1}, v_{2}\right) \in U^{2}$ $L_{v, i}^{k+1}\left(\tilde{\pi}_{\tau}, v_{1}\right) \leq L_{v, i+1}^{k}\left(\pi_{\tau}, v_{2}\right)$, where $\tilde{\pi}_{\tau}$ is generated as in the proof of Proposition 2 with $\pi_{\tau, k}^{*}=\pi_{\tau}$.

Let

$\tilde{X}_{F}:=\{a \in X: \mathcal{P}(a, k)$ for $N$ finite has a feasible timed open-loop control input sequence $\}$.

Note that $\tilde{X}_{F}$ is time-invariant, since the constraints are time-invariant. Further, by A2 and A3, the performance index is finite for every feasible $\pi$ when $N$ is finite.

The stability proof for $\Sigma_{A C L}$ will make use of the following lemma.

\section{Lemma 1}

i. $\mathcal{C}_{F}, A 8$, and $A 9$ imply $0 \in$ Interior $\tilde{X}_{F}$.

ii. A1, A2, A3, A4, and $a \in \tilde{X}_{F}$ imply $\mathcal{P}(a, k)$ for $N$ finite has a solution.

\section{Proof:}

i: From A8 we have, for some $\varepsilon>0, N(\varepsilon) \subset X \times U_{c}$. Let $W(\cdot)$ be the $\mathcal{K}_{\infty}$ function given by property $\mathcal{C}_{F}$. Choose $\lambda>0$ such that $\lambda \leq W^{-1}(\varepsilon)$. If $\|a\| \leq \lambda$, then by A9, $\mathrm{A} 8$, and property $\mathcal{C}_{F}$, there exists a timed open-loop control input sequence which is feasible for $\mathcal{P}(a, k)$. That is, $N(\lambda) \subseteq \tilde{X}_{F}$, and part $i$ is proved.

ii: Follows directly from the hypothesis and the existence proposition.

\section{Proposition 2}

Suppose that (1) satisfies property $\mathcal{C}_{F}$ and that the above assumptions hold. Then:

i. for all $l \geq 0$ and $a \in \tilde{X}_{F}, \lim _{k \rightarrow \infty} x_{k}(l, a)=0$ and $\lim _{t \rightarrow \infty} u(t)=0$.

ii. $x=0$ is the only equilibrium state for $\Sigma_{A C L}$ and it is $U A S$ with region of attraction equal to $\tilde{X}_{F}$.

\section{Proof:}

i: Pick arbitrary $l \geq 0$ and $a \in \tilde{X}_{F}$ and let the solution $x_{k}(l, a)$ of $\Sigma_{A C L}$ be denoted $x_{k}$ for short. In what follows we omit the argument $x_{k}$ unless presence is vital for the proof. From Lemma 1 part ii, $\forall(k, x) \in \mathbb{N} \times \tilde{X}_{F} \phi^{*}(x, k)$ exists. Furthermore, at any $k \geq l$ a feasible solution for $\mathcal{P}\left(x_{k+1}, k+1\right)$ may be extracted from the solution at $k$, cfr. $\tilde{\pi}$ below. Thus, for any $k \geq l$ we have

$$
\begin{aligned}
\phi^{*}\left(x_{k}, k\right)=L_{z, k}\left(x_{k}\right)+L_{z, k+1}\left(z_{k, 1}^{*}\right)+\ldots & \\
+L_{z, k+N-1}\left(z_{k, N-1}^{*}\right)+ & L_{v, 0}^{k}\left(\pi_{\tau, k}^{*}, v_{k, 0}^{*}\right)+\ldots \\
& +L_{v, M-1}^{k}\left(\pi_{\tau, k}^{*}, v_{k, M-1}^{*}\right) .
\end{aligned}
$$

The final state constraint, A5, A6, and A10 imply

$$
\begin{aligned}
\phi^{*}\left(x_{k}, k\right) \geq & L_{z, k}\left(x_{k}\right)+ \\
& \sum_{i \in I_{\alpha-1}} L_{v, i}^{k}\left(\pi_{\tau, k}^{*}, v_{k, i}^{*}\right)+\phi\left(\tilde{\pi} ; z_{k, 1}^{*}\left(x_{k}\right), k+1\right)
\end{aligned}
$$

Next we define $\tilde{\pi}$ and $\alpha . \quad \tilde{\pi}:=\left(\left(\tilde{\tau}_{1}, \ldots, \tilde{\tau}_{M}\right),\left(\tilde{v}_{0}\right.\right.$, $\left.\ldots, \tilde{v}_{M-1}\right)$ ).

Case 1: $\tau_{M}^{*}>\Delta$ :

$$
\begin{gathered}
\alpha:=\min \left\{i \in I_{M}^{+}: \tau_{k, i}^{*}\left(x_{k}\right)>\Delta\right\} \\
\tilde{\tau}_{i}:=\tau_{k, \alpha+i-1}^{*}-\Delta ; \tilde{v}_{i-1}:=v_{k, \alpha+i-2}^{*} ; i \in I_{M-\alpha+1}^{+} .
\end{gathered}
$$

If $\alpha>1$, then

$$
\tilde{\tau}_{i}:=T_{M} ; \tilde{v}_{i-1}:=0 ; i \in\{M-\alpha+2, \ldots, M\} .
$$

Case 2: $\tau_{M}^{*} \leq \Delta$ :

$\alpha:=M, \tilde{\tau}_{i}:=0, i \in I_{M}^{+}$, and $\tilde{v}_{i}:=0, i \in I_{M-1}$.

Next, by optimality and $\Sigma_{A C L}$ :

$$
\phi^{*}\left(x_{k}, k\right)-\phi^{*}\left(x_{k+1}, k+1\right) \geq L_{z, k}\left(x_{k}\right)+\sum_{i \in I_{\alpha-1}} L_{v, i}^{k}\left(\pi_{\tau, k}^{*}, v_{k, i}^{*}\right) .
$$

The sequence $\phi^{*}\left(x_{k}, k\right)$ is non-increasing by nonnegativity of the right-hand side, it is also bounded below (by zero) by A2 and A3, hence it converges as $k \rightarrow \infty$. This implies that the right-hand side converges to zero. By $\mathrm{A} 7, \mathrm{~A} 4$, and continuity of the norm this implies $x_{k} \rightarrow 0$ as $k \rightarrow \infty$, and using the definition of $u(\cdot)$, Eq. (3), it is clear that $u(t) \rightarrow 0$ as $t \rightarrow \infty$. By the arbitrariness of $l$ and $a, i$ follows.

ii: $x=0$ being the only equilibrium follows from the first part. Next we apply Theorem 1 with $\phi^{*}(\cdot, \cdot)$ as the Liapunov function $V(\cdot, \cdot)$. Let $\lambda>0$ be the constant defined in the proof of Lemma 1 part i. Pick $a \in N(\lambda) \subset \tilde{X}_{F}$ and $l \geq 0$. Let $\beta(\|a\|):=(N-1) H_{3}(W(\|a\|))+$ $M H_{2}(W(\|a\|))$, then property $\mathcal{C}_{F}, \mathrm{~A} 9$, and the properties of $H_{3}(\cdot)$ and $H_{2}(\cdot)$ imply $\phi(\pi ; a, l) \leq \beta(\|a\|)$ where $\pi$ is given by $\mathcal{C}_{F}$, clearly $\beta(\cdot) \in \mathcal{K}_{+}$. Now, by optimality $\phi^{*}(a, l) \leq \phi(\pi ; a, l) \leq \beta(\|a\|)$. Let $\alpha(\cdot)=H_{4}(\cdot)$, then, by $\mathrm{A} 2$ and $\mathrm{A} 3, \phi^{*}(a, l) \geq \alpha(\|a\|)$. Let $\gamma(\cdot)=H_{4}(\cdot)$, then, by $\mathrm{A} 2$ and $\mathrm{A} 3, \phi^{*}(a, l)-\phi^{*}\left(x_{l+1}(l, a), l+1\right) \geq \gamma(\|a\|)$. In conclusion we see that conditions (a), (b), and (c) of Theorem 1 are satisfied, and hence the origin is UAS for $\Sigma_{A C L} \cdot \tilde{X}_{F}$ is the region of attraction by part $i$.

Now, we proceed to analysis of $\Sigma_{C L}$.

\section{Definition 2 ( $\epsilon$-UAS)}

Given the system

$\dot{x}(t)=f\left(x(t), x\left(t_{k}\right), t\right), x\left(t_{k}\right) \in \tilde{X} \subseteq R^{n}, t \in\left[t_{k}, t_{k+1}\right), k \geq 0$,

where $f: R^{n} \times \tilde{X} \times R \rightarrow R^{n}$ is sufficiently smooth for existence and uniqueness of a solution, and $f(0,0, t)=0$ for all $t \geq 0$. Let $x(t)$ denote the solution of Eq. (5) for $t \geq t^{0}$ given $x\left(t^{0}\right)$ and $x\left(\left\lfloor\frac{t^{0}}{\Delta}\right\rfloor \Delta\right)$. The origin is said to be $\epsilon$-uniformly asymptotically stable $(\epsilon$-UAS) if:

1.

$$
\begin{aligned}
& \forall x\left(\left\lfloor\frac{t^{0}}{\Delta}\right\rfloor \Delta\right) \in \tilde{X}, t^{0} \geq 0, \varepsilon>\epsilon, \exists \delta(\varepsilon)>0, \\
& \text { s.t. } x\left(t^{0}\right) \in N(\delta) \Rightarrow x(t) \in N(\varepsilon) \quad \forall t \geq t^{0} .
\end{aligned}
$$


If this is satisfied we say that the origin is $\epsilon$-US for Eq. (5). 2. It is uniformly convergent (UC) in the following sense:

$$
\begin{gathered}
\exists r>0, \text { s.t } \forall x\left(\left\lfloor\frac{t^{0}}{\Delta}\right\rfloor \Delta\right) \in \tilde{X}, t^{0} \geq 0, \sigma>0, \exists T(\sigma) \geq 0, \\
\text { s.t. } x\left(t^{0}\right) \in N(r) \Rightarrow x(t) \in N(\sigma) \forall t \geq t^{0}+T .
\end{gathered}
$$

\section{Theorem 2}

Suppose $U$ compact, and let $K:=\max _{u \in U}\|u\|$. Also, suppose

$$
e^{\|A\| \Delta} K \Delta\|B\|<\min \left\{r_{A C L}, p(\epsilon)\right\},
$$

where $r_{A C L}>0$ and $p(\cdot) \in \mathcal{K}_{+}$are known [7]. Combined with assumptions $A 1-A 10$ and property $\mathcal{C}_{F}$ this implies that the origin is $\epsilon-U A S$ for $\Sigma_{C L}$.

Proof:

$f(\cdot, \cdot, \cdot)$ in Def. 2 is given by: $f\left(x(t), x\left(t_{k}\right), t\right):=A x(t)+$ $B v_{\lfloor t / \Delta\rfloor}^{*}\left(t-\left\lfloor\frac{t}{\Delta}\right\rfloor \Delta ; x\left(t_{k}\right)\right)$ and $\tilde{X}:=\tilde{X}_{F} \cdot f(0,0, t)=0$, $\forall t \geq 0$ by optimality and since $A 0=0$.

$\epsilon$-US: Pick $t^{0} \geq 0, x\left(\left\lfloor\frac{t^{0}}{\Delta}\right\rfloor \Delta\right) \in \tilde{X}_{F}$, and $\varepsilon>\epsilon$. For $t \in$ $\left[t^{0},\left\lceil\frac{t^{0}}{\Delta}\right\rceil \Delta\right]$ we have:

$$
\begin{gathered}
\|x(t)\|=\left\|e^{A\left(t-t^{0}\right)} x\left(t^{0}\right)+\int_{t^{0}}^{t} e^{A(t-\sigma)} B u(\sigma) \mathrm{d} \sigma\right\|, \\
\leq e^{\|A\| \Delta}\left(\left\|x\left(t^{0}\right)\right\|+K \Delta\|B\|\right) .
\end{gathered}
$$

When there are no perturbations (i.e. nominally) it can be shown $([7])$ that for $t \in\left[t_{k}, t_{k+1}\right]$ :

$$
\|x(t)\| \leq H\left(\| x_{k}||\right), H(\cdot) \in \mathcal{K}_{\infty},
$$

where $H(\cdot)$ is given by $\|A\|,\|B\|, \Delta$, and some of the functions in the assumptions and property $\mathcal{C}_{F}$. From this we see that if we, with $H^{-1}(\varepsilon)$, associate an $\varepsilon_{A C L}:=$ $H^{-1}(\varepsilon)$ for $\Sigma_{A C L}$ and ensure by restricting $\left\|x\left(t^{0}\right)\right\|$ that $\left\|x\left(\left\lceil\frac{t^{0}}{\Delta}\right\rceil \Delta\right)\right\| \leq \delta_{A C L}=: p(\varepsilon)$ where $\delta_{A C L}$ corresponds to $\varepsilon_{A C L}$ we will, by the property of $H(\cdot)$ and (uniform) stability of $\Sigma_{A C L}$, be guaranteed that $\|x(t)\| \leq \varepsilon$, for all $t \geq t^{0}$. By the hypothesis this can be done by restricting $x\left(t^{0}\right)$ to $N(\delta)$ where $\delta(\varepsilon):=\frac{p(\varepsilon)}{e^{|| A|| \Delta}}-K \Delta\|B\|>0$.

$U C$ : This follows in the same manner as for $\epsilon$-US. By the hypothesis there is an initial set, in which $x\left(t^{0}\right)$ can be picked, with radius $r:=\frac{r_{A C L}}{e^{\|A\| \Delta}}-K \Delta\|B\|>0$ which ensures that $\left\|x\left(\left\lceil\frac{t^{0}}{\Delta}\right\rceil \Delta\right)\right\| \leq r_{A C L}$, where $r_{A C L}$ is the radius of the initial set for $\Sigma_{A C L}$. Now, given some $\sigma>0$ we let $\sigma_{A C L}:=H^{-1}(\sigma)$, and $T:=T_{A C L} \Delta+\Delta$, where $T_{A C L}$ corresponds to $\sigma_{A C L}$. This implies that $\|x(t)\| \leq \sigma$ for all $t \geq t^{0}+T$ by the property of $H(\cdot)$ and since the state of $\Sigma_{A C L}$ is (uniformly) convergent to the origin (starting within $\left.N\left(r_{A C L}\right)\right)$.

\section{Discussion}

Our control approach is limited to linear time-invariant systems, a natural extension would be to consider linear time-varying systems, or even nonlinear systems. For nonlinear systems, the controllability property, $\mathcal{C}_{F}$, will in general be difficult to verify [4]. Including the possibility for a delayed sampled-state measurement would also increase the applicability of the approach. In our analysis we have assumed that the global optima of the optimization problems are found. Although we have proved existence of global solutions, the problem of finding them is not investigated.

\section{Conclusions}

An MPC strategy for control of a class of constrained sampled-data hybrid systems is proposed, and so called $\epsilon$-UAS of the origin for the closed-loop system is established.

\section{References}

[1] Rajeev Alur, Thomas A. Henzinger, and Eduardo D. Sontag, Eds., Hybrid Systems III: Verification and Control, number 1066 in LNCS. Springer, 1996.

[2] Sebastian Engell and Stefan Kowalewski, "Discrete Events and Hybrid Systems in Process Control", in CPC-V. Tahoe City, Cal, USA, January 1996.

[3] J. B. Rawlings, E. S. Meadows, and K. R. Muske, "Nonlinear Model Predictive Control: A Tutorial and Survey", in Preprints IFAC Symposium ADCHEM, Kyoto, Japan, 1994.

[4] S. S. Keerthi and E. G. Gilbert, "Optimal InfiniteHorizon Feedback Laws for a General Class of Constrained Discrete-Time Systems: Stability and Moving-Horizon Approximations", Journal of Optimization Theory and Applications, vol. 57, no. 2, pp. 265-93, May 1988.

[5] D. Q. Mayne, "Nonlinear Model Predictive Control: An Assessment", in CPC-V. Tahoe City, Cal, USA, January 1996.

[6] Edward E. Meadows, Michael A. Henson, John W. Eaton, and James B. Rawlings, "Receding horizon control and discontinuous state feedback stabilization", Int. J. Control, vol. 5, pp. 1217-29, 1995.

[7] Olav Slupphaug, "Model Predictive Control for a class of Hybrid Systems", Tech. Rep. 96-46-W, Norwegian University of Science and Technology, Department of Engineering Cybernetics, 1996.

[8] S. S. Keerthi and E. G. Gilbert, "An Existence Theorem for Discrete-Time Infinite-Horizon Optimal Control Problems", IEEE Transactions on Automatic Control, vol. 30, no. 9, pp. 907-9, September 1985.

[9] Jaroslav Doležal, "Existence of Optimal Solutions in General Discrete Systems", Kybernetika, vol. 11, no. 4, pp. 301-12, 1975.

[10] E. D. Sontag, Mathematical Control Theory, Number 6 in Texts in Applied Mathematics. Springer, 1990 . 